\title{
Polysaccharide from Fuzi protects against Ox-LDL-induced calcification of human vascular smooth muscle cells by increasing autophagic activity
}

\author{
LIZHEN LIAO ${ }^{1 *}$, XIAODONG ZHUANG ${ }^{2 *}$, WEIDONG LI ${ }^{1}$, QIBIAO SU ${ }^{1}$, JIE ZHAO $^{1}$ and YING LIU ${ }^{1}$ \\ ${ }^{1}$ Guangdong Pharmaceutical University, Guangzhou Higher Education Mega Center, Guangzhou, \\ Guangdong 510006; ${ }^{2}$ Department of Cardiology, The First Affiliated Hospital of Sun Yat-sen University, \\ Guangzhou, Guangdong 510080, P.R. China
}

Received March 21, 2017; Accepted September 1, 2017

DOI: $10.3892 / \mathrm{mmr} .2018 .8488$

\begin{abstract}
Polysaccharide from Fuzi (FPS) is a water-soluble polysaccharide isolated from the traditional Chinese herbal medicine Fuzi. It has been demonstrated to protect hepatocytes against ischemia-reperfusion injury through its potent antioxidant effects, and to attenuate starvation-induced cytotoxicity in H9c2 cells by increasing autophagic activity. In the present study, Alizarin Red S staining was used to detect mineral deposition and reverse transcription-quantitative polymerase chain reaction was used to detect the core binding factor $\alpha 1$ and smooth muscle $22 \alpha$ mRNA expression. To analyze autophagic activity, western blotting was used to detect microtubule-associated protein 1A/1B light chain 3 and nucleoporin P62 expression. In addition, green fluorescent protein-LC3 dots-per-cell was observed by fluorescence microscopy. It was demonstrated that oxidized low-density lipoprotein (Ox-LDL) could increase the calcification of human vascular smooth muscle cells (VSMCs) in a concentration-dependent manner, and that FPS treatment had a significant protective effect against Ox-LDL-induced calcification of human VSMCs. Furthermore, FPS treatment alleviated the Ox-LDL-induced downregulation of autophagic activity, and the protective effect of FPS on Ox-LDL-induced calcification was attenuated by the autophagy inhibitor 3-methyladenine. In conclusion, the present study demonstrated for the first time to the best of the authors' knowledge that FPS can protect against Ox-LDL-induced vascular calcification in human VSMCs, and that this likely occurs via the activation of autophagy. This supports the
\end{abstract}

Correspondence to: Dr Ying Liu, Guangdong Pharmaceutical University, Guangzhou Higher Education Mega Center, 280 Wai Huan Dong Road, Guangzhou, Guangdong 510006, P.R. China E-mail: tonyandanna@163.com

\section{${ }^{*}$ Contributed equally}

Key words: polysaccharide from Fuzi, human vascular smooth muscle cell, calcification, autophagy hypothesis that autophagy may be an endogenous protective mechanism counteracting vascular calcification, and that FPS may be used as a potential therapeutic for vascular calcification.

\section{Introduction}

Vascular calcification is an important medical issue due to the close association between the degree of vascular calcification and the severity of various clinical diseases, including atherosclerosis (1), diabetes mellitus (2) and chronic kidney disease (3). In vessel calcification, vascular smooth muscle cells (VSMCs) serve an integral mediatory role by undergoing differentiation into osteoblast-like cells and generating matrix vesicles, serving as nidi for calcium-phosphate deposition in the vessel wall (4). During this process, upregulation of bone-associated genes, such as core binding factor $\alpha 1$ (CBFA1) (5), and downregulation of smooth muscle cell-associated marker genes, such as smooth muscle $22 \alpha$ (SM22 $\alpha$ ) (6), can be observed.

Oxidized low-density lipoproteins (Ox-LDL) are crucial in the progression of atherosclerosis, and promote osteogenic differentiation and calcification of VSMCs (7). In addition, the catabolic process of autophagy, which can occur as an adaptive response to cell stress, has been demonstrated to limit VSMC calcification (8). Accumulating evidence has demonstrated that the enhancement of autophagy by different types of medicinal molecules may serve as a potential therapeutic approach for Ox-LDL-induced pathological states $(9,10)$.

Numerous polysaccharide-containing Chinese herbal medicines are capable of exerting a wide variety of pharmacological effects, including cardioprotection $(11,12)$. One such polysaccharide has previously been extracted from Radix Aconitum carmichaelii Praeparata (also known as Fuzi), and is designated as polysaccharide from Fuzi (FPS) (13). Previously, it was demonstrated that FPS pretreatment protected hepatocytes against ischemia-reperfusion injury through its potent anti-oxidative effects and necrosis attenuation (14). Furthermore, it was demonstrated that FPS increased autophagic activity to protect against starvation-induced cytotoxicity in H9c2 cells (15). Therefore, it can be hypothesized that FPS may also have protective effects against calcification in human VSMCs during Ox-LDL-induced stress, via autophagy upregulation. 


\section{Materials and methods}

Cell culture. All cell culture reagents were purchased from Sigma-Aldrich (Merck KGaA, Darmstadt, Germany). Written informed consent was obtained from patients and approval was received from the Ethics Committee of the First Affiliated Hospital of Sun Yat-sen University (Guangzhou, China). The work described in the present study was conducted in accordance with The Code of Ethics of the World Medical Association (www.wma.net/). VSMCs were isolated from human femoral arteries (4 patients, $45.4 \pm 4.6$ years old, 2 male and 2 female, recruited from Guangzhou, China, from June 2016 to May 2017) using the explant method described previously (16). VSMCs were maintained in Dulbecco's modified Eagle's medium (DMEM) supplemented with $10 \%$ fetal bovine serum at $37^{\circ} \mathrm{C}$ in $5 \% \mathrm{CO}_{2}$. Both DMEM and fetal serum were purchased from Sigma-Aldrich (Merck KGaA). Cells between passages 3 and 6 were used in this study. To induce calcification, growth medium was replaced with osteogenic DMEM, which was supplemented with $10 \mathrm{mM} \beta$-glycerophosphate, which was purchased from Sigma-Aldrich (Merck KGaA).

Ox-LDL preparations. LDLs (1.019-1.063 g/ml) were separated from human plasma by sequential density gradient ultracentrifugation $\left(145,000 \mathrm{x} \mathrm{g}\right.$ at $10^{\circ} \mathrm{C}$ for $\left.20 \mathrm{~h}\right)$. The LDL fraction was dialyzed against PBS (pH 7.4) at $4^{\circ} \mathrm{C}$ for $24 \mathrm{~h}$. Ox-LDL was prepared by incubation of LDL with $5 \mathrm{mM}$ copper sulfate (Sigma-Aldrich, Merck KGaA) at $37^{\circ} \mathrm{C}$ for $3 \mathrm{~h}$, and was subsequently sterilized by passaging through a $0.22-\mu \mathrm{m}$ filter. Cells were treated with Ox-LDL $(10,50$ or $100 \mu \mathrm{g} / \mathrm{ml})$ for 14 days $\left(37^{\circ} \mathrm{C}\right.$, treated once every two days).

Mineralization assay. Mineral deposition in cultured VSMCs was assessed by Alizarin Red S staining as previously described (17). Calcified VSMCs $\left(2 \times 10^{6}\right)$ were fixed in $4 \%$ formaldehyde for $10 \mathrm{~min}$ at room temperature and exposed to $2 \%$ Alizarin Red S (pH 4.2) for 5 min at room temperature. Subsequently, cells were washed with deionized water to remove excess dye and imaged using an inverted phase contrast microscope. For the quantification of Alizarin Red S staining, $10 \%$ formic acid was used to elute the Alizarin Red S dye, and the absorbance at $405 \mathrm{~nm}$ was determined with a microplate reader and normalized to the protein content. For quantification of calcium content, cells were washed with PBS and decalcified with $0.6 \mathrm{M}$ hydrogen chloride for $24 \mathrm{~h}$. The calcium content of the cultures was determined colorimetrically using the O-cresolphthalein complexone method and normalized to protein content as previously described (18).

Reverse transcription-quantitative polymerase chain reaction $(R T-q P C R)$. Total RNA was isolated from cultured VSMCs using TRIzol reagent (Invitrogen; Thermo Fisher Scientific, Inc., Waltham, MA, USA), and was reverse transcribed using an avian myeloblastosis virus Reverse Transcriptase (Roche Diagnostics GmbH, Mannheim, Germany) according to the manufacturer's protocol. The thermocycling conditions were $37^{\circ} \mathrm{C}$ for $1 \mathrm{~h}$ and then $94^{\circ} \mathrm{C}$ for $10 \mathrm{~min}$. RT-qPCR was performed using a SYBR ${ }^{\circledR}$-Green PCR Master Mix (Applied Biosystems; Thermo Fisher Scientific, Inc.) in a StepOne Real Time PCR system (Applied Biosystems; Thermo Fisher Scientific, Inc.).
The primers used for RT-qPCR were as follows: CBFA1 forward, 5'-CCGTGGCCTTCAAGGTTGT-3' and reverse, 5'-TTCATAACAGCGGGAGGCATTTC-3'; SM22 $\alpha$ forward, 5'-TTGGATCCGACATGGCCAACAAG-3' and reverse, 5'-GCCCTAAGCTTTCTAACTGATGATCT-3'; and $\beta$-actin forward, 5'-CCAGCTCACCATGGATGATG-3' and reverse, 5'-GAGCCGTTGTCGACGACG-3'. Values were normalized to those of $\beta$-actin. The results were calculated using the comparative threshold cycle (Cq) method (19).

Treatments. In all assays human VSMCs were cultured in osteogenic medium at $37^{\circ} \mathrm{C}$ with $5 \% \mathrm{CO}_{2}$ for 14 days. Experiment 1 (Fig. 1) human VSMCs were treated with Ox-LDL (0, 10, 50 and $100 \mu \mathrm{g} / \mathrm{ml}$ ). In experiment 2 (Fig. 2) cells were treated with 0 or $50 \mu \mathrm{g} / \mathrm{ml}$ Ox-LDL and different concentrations of FPS $(0,10,100$ or $1,000 \mu \mathrm{g} / \mathrm{ml})$. Experiment 3 (Fig. 3) was performed with 0 or $50 \mu \mathrm{g} / \mathrm{ml}$ Ox-LDL and $1,000 \mu \mathrm{g} / \mathrm{ml}$ FPS. In experiment 4 (Fig. 4) human VSMCs with 0 or $50 \mu \mathrm{g} / \mathrm{ml}$ Ox-LDL, $1,000 \mu \mathrm{g} / \mathrm{ml} \mathrm{FPS} \mathrm{and} 5 \mathrm{mM} 3 \mathrm{MA}$.

Western blot analysis. Cells $\left(1 \times 10^{7}\right)$ were plated in $100-\mathrm{mm}$ diameter Petri dishes and were treated as indicated above following $70-80 \%$ confluency. Following treatment, cells were harvested and resuspended in ice-cold cell lysis buffer (Sigma-Aldrich, Merck KGaA), and the homogenate was centrifuged at $10,000 \mathrm{x}$ g for $15 \mathrm{~min}$ at $4^{\circ} \mathrm{C}$. The total supernatant protein concentration was measured using a bicinchoninic acid protein assay kit. Total protein (50 $\mu \mathrm{g})$ from each sample was separated by $15 \%$ sodium dodecyl sulfate-polyacrylamide gel electrophoresis and transferred to a polyvinylidene difluoride membrane. The membrane was blocked with $5 \%$ fat-free dry milk in TBS with Tween-20 for $1.5 \mathrm{~h}$ at room temperature, followed by incubation with primary antibodies against microtubule-associated protein 1A/1B light chain 3 (LC3B), nucleoporin P62 and GAPDH (cat. nos. 2775, 5114 and 5014; 1:1,000; Cell Signaling Technology, Inc., Danvers, MA, USA) overnight with gentle agitation at $4^{\circ} \mathrm{C}$. The next day, the membranes were washed with PBS and subsequently incubated with horseradish peroxidase-conjugated secondary antibodies (cat. no. 7075; 1:1,000; Cell Signaling Technology, Danvers, MA, USA) for $1.5 \mathrm{~h}$ at room temperature. Following three washes with TBS with Tween-20, membranes were developed using an enhanced chemiluminescence kit (Applygen Technologies, Inc., Beijing, China) and exposed to X-ray films. ImageJ software (1.48u version; National Institutes of Health, Bethesda, MD, USA) was used to quantify the protein expression levels.

Cell transfections and green fluorescent protein (GFP)-LC3 dot-per-cell quantification. Cells were transfected with pEGFP-LC3 plasmids (cat. no. 24920; Addgene, Cambridge, MA, USA) using Lipofectamine ${ }^{\mathrm{TM}}$ LTX (cat. no. 11668019; Thermo Fisher Scientific, Inc.) reagent as previously described (20); a minimum of $40 \%$ transfection efficiency was achieved and GFP-LC3-transfected cells were visualized using a fluorescence microscope.

Statistical analysis. All assays were performed $\geq 4$ times and the values were expressed as the mean \pm standard error. Statistical analysis of between-group differences was performed by one-way 
A

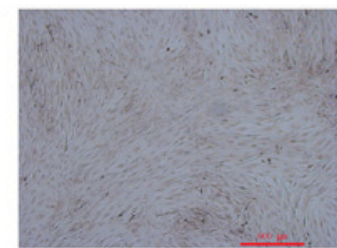

Ox-LDL $(\mu \mathrm{g} / \mathrm{ml})$

B

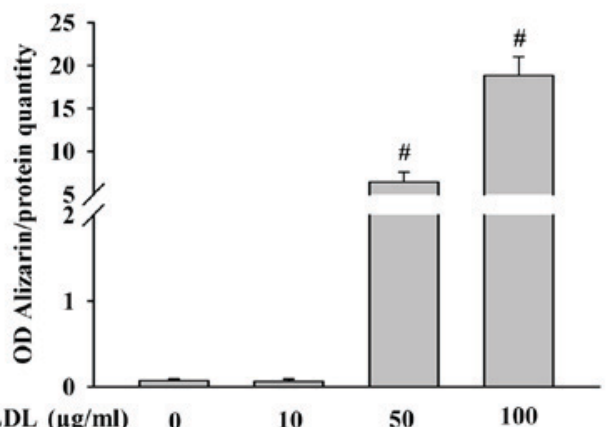

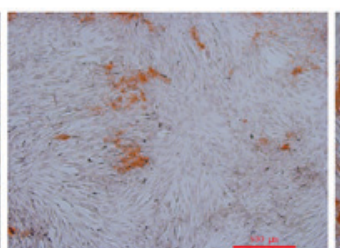

$\mathbf{5 0}$

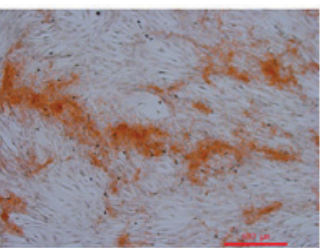

100

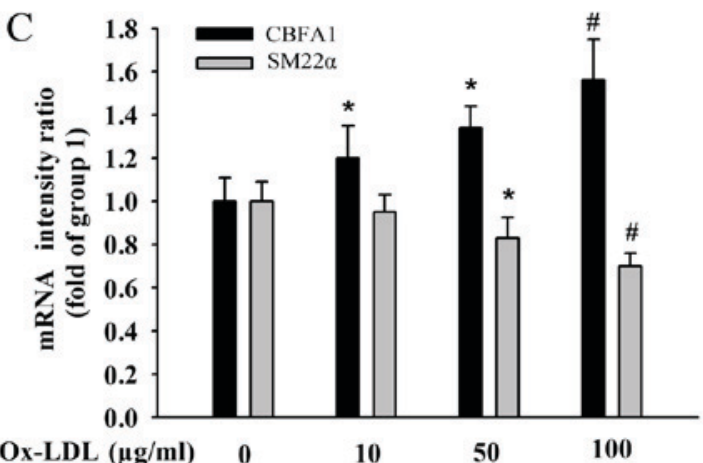

Ox-LDL $(\mu \mathrm{g} / \mathrm{ml}) \quad 0$

Figure 1. Human VSMCs were cultured in osteogenic medium with or without Ox-LDL (10, 50 and $100 \mu \mathrm{g} / \mathrm{ml}$ ) for $14 \mathrm{days}$. Representative images of VSMCs stained with (A) Alizarin Red S to assess mineralization and (B) quantification of it. (C) CBFA1 and SM22 $\alpha$ mRA expression levels as determined by RT-qPCR. Data are expressed as the mean \pm standard error $(n=4)$. Group 1 represents the control group. ${ }^{*} \mathrm{P}<0.05$ vs. group 1 ; ${ }^{\#} \mathrm{P}<0.001$ vs. group 1 . VSMCs, vascular smooth muscle cells; Ox-LDL, oxidized low-density lipoproteins; CBFA1, core binding factor $\alpha 1$; SM22 $\alpha$, smooth muscle 22 $\alpha$; OD, optical density.
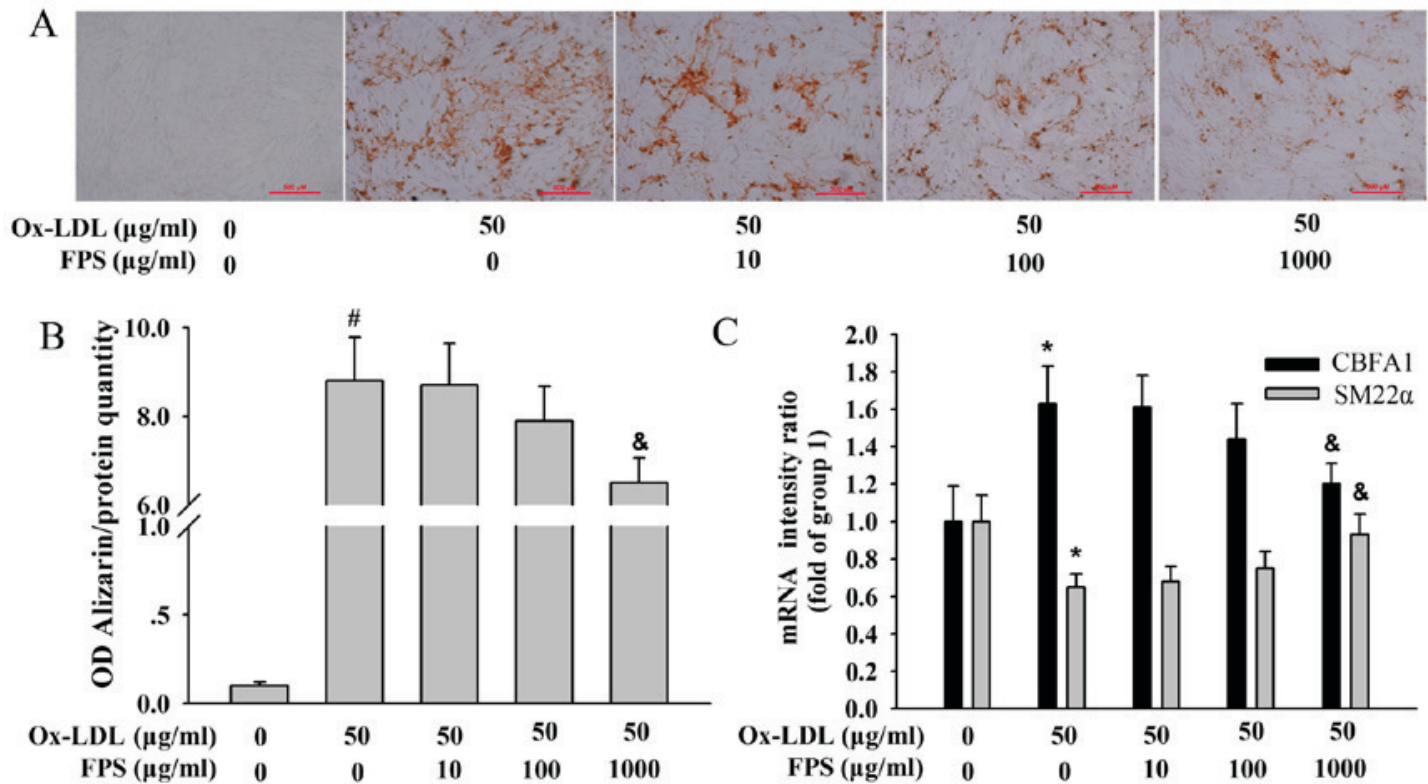

Figure 2. Human VSMCs were cultured in osteogenic medium with or without $50 \mu \mathrm{g} / \mathrm{ml} \mathrm{Ox-LDL}$ and different concentrations of FPS $(0,10,100$ or $1,000 \mu \mathrm{g} / \mathrm{ml})$ for 14 days. Representative images of VSMCs stained with (A) Alizarin Red S to assess mineralization and (B) quantification of it. (C) CBFA1 and SM22 $\alpha$ mRNA expression levels as determined by RT-qPCR. Data are expressed as the mean \pm standard error (n=4). In group 1 human VSMCs were cultured in osteogenic medium without Ox-LDL or FPS. In group 2 human VSMCs were cultured in osteogenic medium with $50 \mu \mathrm{g} / \mathrm{ml}$ Ox-LDL without FPS. ${ }^{*} \mathrm{P}<0.05$ vs. group 1; ${ }^{\text {P }}<0.001$ vs. group $1 ;{ }^{\&} \mathrm{P}<0.05$ vs. group 2 . VSMCs, vascular smooth muscle cells; Ox-LDL, oxidized low-density lipoproteins; FPS, polysaccharide from Fuzi; CBFA1, core binding factor $\alpha 1$; SM22 $\alpha$, smooth muscle 22 $\alpha$; OD, optical density.

analysis of variance with a Bonferroni post hoc test with using SPSS 16.0 software (SPSS, Inc., Chicago, IL, USA). P<0.05 was considered to indicate a statistically significant difference.

\section{Results}

Ox-LDL increases human VSMC calcification in a concentration-dependent manner. Human VSMCs were treated with 10,50 or $100 \mu \mathrm{g} / \mathrm{ml}$ Ox-LDL for 14 days to determine the effect of Ox-LDL on vascular calcification. Alizarin Red S staining was used to assess mineralization, which is positively associated with VSMC mineralization. As illustrated in Fig. 1A and B, Ox-LDL increased human VSMC calcification in a concentration-dependent manner, particularly in the 50 and $100 \mu \mathrm{g} / \mathrm{ml}$ Ox-LDL treatment groups. Additionally, RT-qPCR revealed that Ox-LDL increased CBFAl mRNA 
A

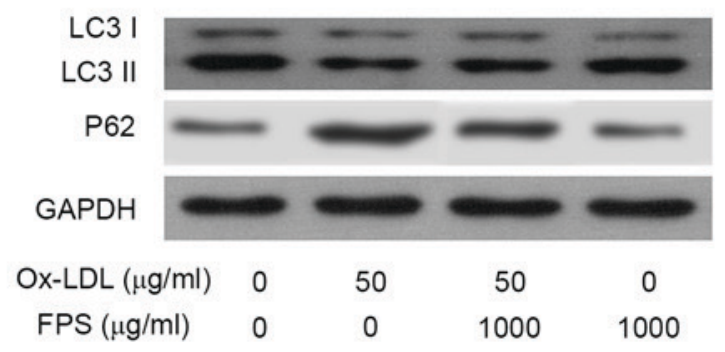

C

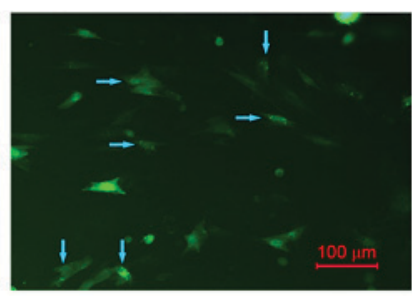

Ox-LDL $(\mu \mathrm{g} / \mathrm{ml}) \quad 0$

FPS $(\mu \mathrm{g} / \mathrm{ml}) \quad 0$

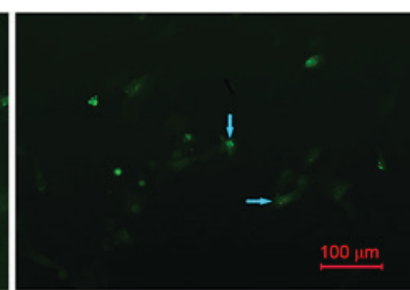

50

0
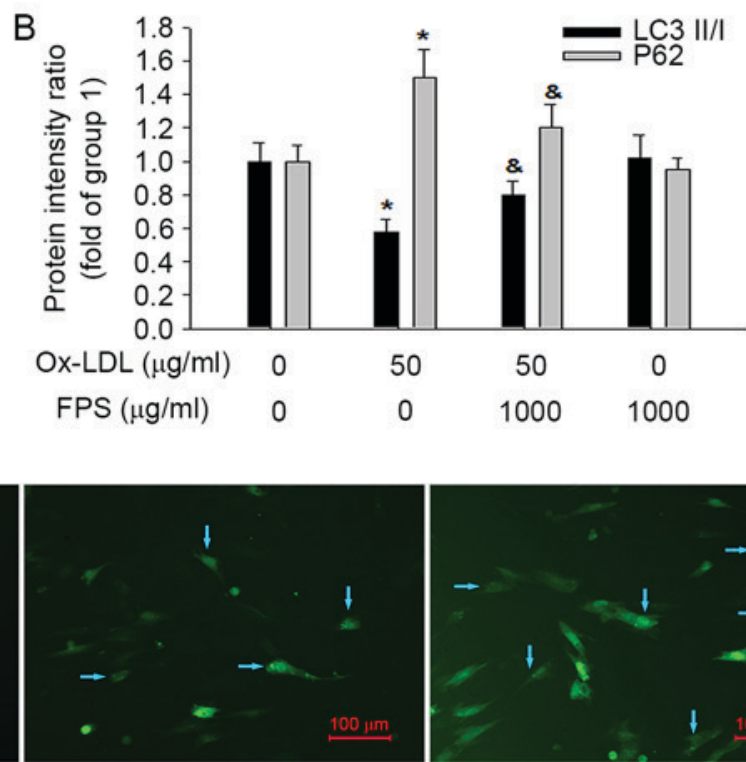

50

1000

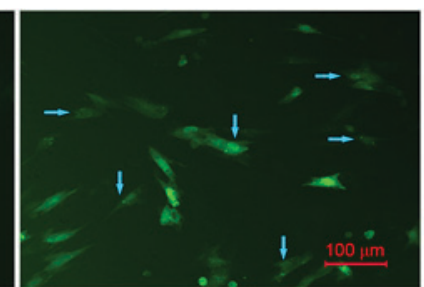

0

1000

Figure 3. Human VSMCs were cultured in osteogenic medium with or without $50 \mu \mathrm{g} / \mathrm{ml}$ Ox-LDL and $1,000 \mu \mathrm{g} / \mathrm{ml} \mathrm{FPS} \mathrm{for} 14$ days. Representative images of (A) LC3-I, LC3-II, P62 and GAPDH proteins and (B) quantification of the relative LC3-II/LC3-I ratio and relative P62 protein expression levels, as measured by western blotting. (C) Representative images of GFP-LC3 dots after the indicated treatments. Blue arrows represent VSMCs expressing GFP-LC3 dots. Data are expressed as the mean \pm standard error $(n=4)$. Group 1, human VSMCs were cultured in osteogenic medium without Ox-LDL or FPS. In group 2 human VSMCs were cultured in osteogenic medium with $50 \mu \mathrm{g} / \mathrm{ml}$ Ox-LDL without FPS. ${ }^{*} \mathrm{P}<0.05$ vs. group 1 ; ${ }^{\&} \mathrm{P}<0.05$ vs. group 2 . VSMCs, vascular smooth muscle cells; Ox-LDL, oxidized low-density lipoproteins; FPS, polysaccharide from Fuzi; GFP, green fluorescent protein; LC3, microtubule-associated protein 1A/1B light chain 3; OD, optical density.

expression in a concentration-dependent manner, whereas it decreased $S M 22 \alpha$ mRNA expression (Fig. 1C), further confirming that Ox-LDL increased human VSMC calcification. For the subsequent experiments, $50 \mu \mathrm{g} / \mathrm{ml}$ Ox-LDL treatment for 14 days was selected as this induced VSMC calcification and increased Alizarin Red S staining along with increasing CBFA1 mRNA expression and decreasing SM22 $\alpha$ mRNA expression.

FPS treatment attenuates $O x-L D L$-induced human VSMC calcification in a concentration-dependent manner. Various concentrations of FPS were added to the cells to determine its protective effect against vascular calcification induced by Ox-LDL. As demonstrated in Fig. 2A, FPS treatment attenuated the Ox-LDL-induced increase in Alizarin Red $\mathrm{S}$ staining in human VSMCs, particularly in the $1,000 \mu \mathrm{g} / \mathrm{ml}$ FPS-treatment group (Fig. 2B). Furthermore, the CBFAl and $S M 22 \alpha$ mRNA changes induced by Ox-LDL were significantly attenuated by $1,000 \mu \mathrm{g} / \mathrm{ml}$ FPS (Fig. 2C), suggesting that FPS treatment protected against Ox-LDL-induced calcification in human VSMCs.

FPS treatment alleviates the $O x-L D L$-induced downregulation of autophagy activity. It was also investigated whether autophagy was upregulated or downregulated following 14 days of Ox-LDL treatment, and whether FPS treatment affected autophagic activity. The protein expression levels of LC3-I, LC3-II, P62 and GAPDH were measured by western blotting. As presented in Fig. 3A and B, following $50 \mu \mathrm{g} / \mathrm{ml}$ Ox-LDL-treatment for 14 days, the LC3-II/LC3-I ratio was decreased and P62 protein expression was increased, indicating that autophagy was inhibited in the vascular calcification progress. Notably, treatment with $1,000 \mu \mathrm{g} / \mathrm{ml}$ FPS attenuated the aforementioned changes in LC3-II/LC3-I ratio and P62 protein intensity induced by Ox-LDL, suggesting that FPS treatment protected against the Ox-LDL-induced downregulation of autophagic activity. Additionally, no change in autophagic activity was detected in native FPS-treated cells compared with the control group of untreated cells. As demonstrated in Fig. 3C, GFP-LC3 puncta, as indicators of autophagosomes, were also used to monitor autophagic activity. A greater number of GFP-LC3 dots in each VSMC represents increased autophagy. Compared with the control group, fewer GFP-LC3 dots were detected in the Ox-LDL treatment group. When $1,000 \mu \mathrm{g} / \mathrm{ml}$ FPS was added, the Ox-LDL-induced reduction in the number of GFP-LC3 dots was attenuated. This further confirmed that FPS treatment alleviated the Ox-LDL-induced downregulation of autophagy.

Autophagy inhibition by 3-methyladenine (3MA) decreases the protective effect of FPS against VSMC calcification. Whether increased autophagy activity contributed to FPS-mediated protection against vascular calcification was also investigated. Since 3MA is commonly used as a pharmacological agent for the inhibition of autophagy in vitro, VSMCs were treated with 5 mM3MA (Sigma-Aldrich, Merck KGaA) to inhibit autophagy, and the effects on FPS-mediated protection against vascular calcification were assessed. As presented in Fig. 4A and B, when 3MA was added, the protective effect of FPS against VSMC calcification was also inhibited. Additionally, 3MA treatment decreased the LC3-II/LC3-I ratio and increased the $\mathrm{P} 62$ protein level, indicating that autophagic activity was 


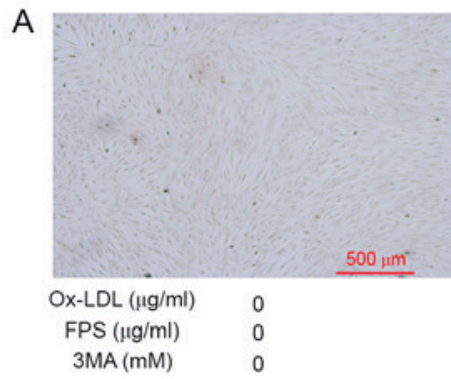

B

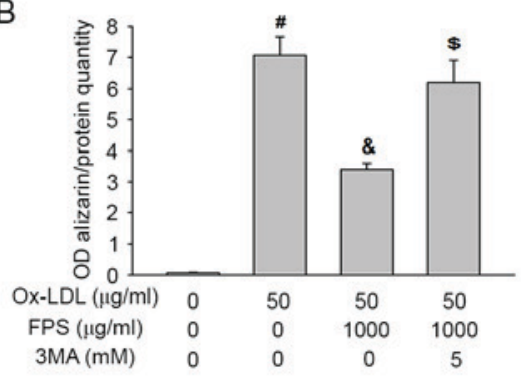

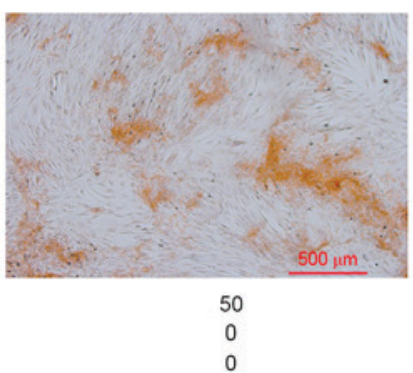

C
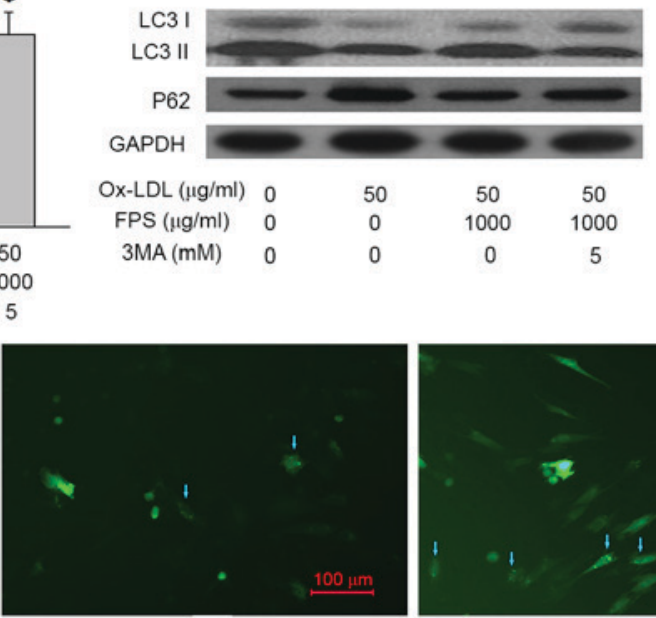

Ox-LDL $(\mu \mathrm{g} / \mathrm{ml})$ FPS $(\mu \mathrm{g} / \mathrm{ml})$ $3 \mathrm{MA}(\mathrm{mM})$

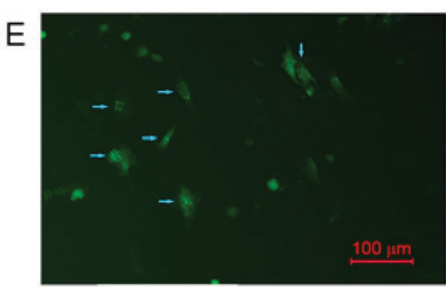

50

0

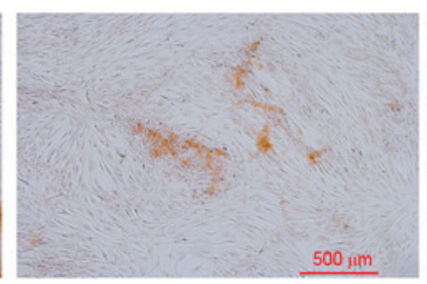

50

1000
0
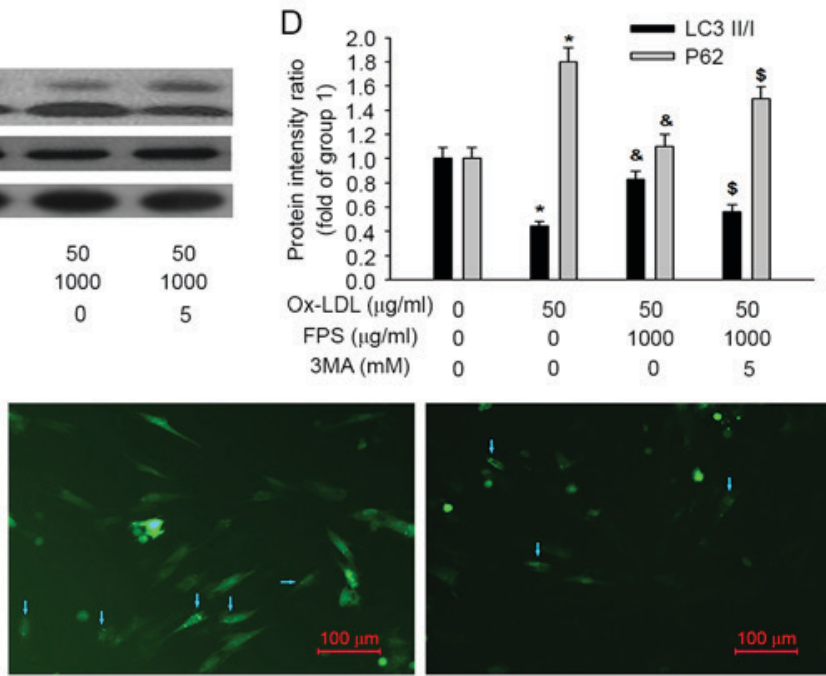

50

1000

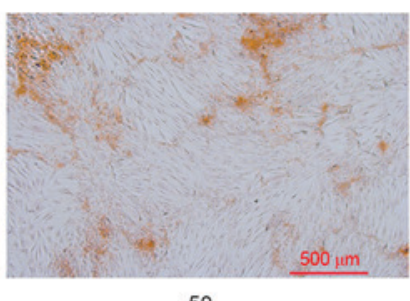

1000

5

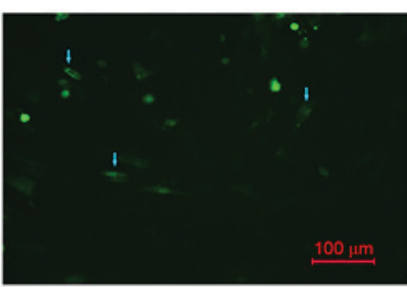

50

1000

Figure 4. Human VSMCs were cultured in osteogenic medium with or without $50 \mu \mathrm{g} / \mathrm{ml}$ Ox-LDL, $1,000 \mu \mathrm{g} / \mathrm{ml}$ FPS and 5 mM $3 \mathrm{MA}$ for 14 days. Representative images of VSMCs stained with (A) Alizarin Red S to assess mineralization and (B) quantification of it. Representative images of (C) LC3-I, LC3-II, P62 and GAPDH proteins and (D) quantification of the relative LC3-II/LC3-I ratio and relative P62 protein expression levels, as assessed by western blotting. (E) Representative images of GFP-LC3 dots after the indicated treatments. Blue arrows represent VSMCs expressing GFP-LC3 dots. Data are expressed as the mean \pm standard error $(n=4)$. In group 1 human VSMCs were cultured in osteogenic medium without Ox-LDL or FPS. Group 2, human VSMCs were cultured in osteogenic medium with $50 \mu \mathrm{g} / \mathrm{ml} \mathrm{Ox-LDL}$ without FPS. In group 3 human VSMCs were cultured in osteogenic medium with $50 \mu \mathrm{g} / \mathrm{ml}$ Ox-LDL and $1,000 \mu \mathrm{g} / \mathrm{ml} \mathrm{FPS.}{ }^{\#} \mathrm{P}<0.001$ vs. group 1 ; ${ }^{*} \mathrm{P}<0.05$ vs. group $1 ;{ }^{\&} \mathrm{P}<0.05$ vs. group 2 ; ${ }^{\$} \mathrm{P}<0.05$ vs. group 3 . VSMCs, vascular smooth muscle cells; Ox-LDL, oxidized low-density lipoproteins; FPS, polysaccharide from Fuzi; GFP, green fluorescent protein; LC3, microtubule-associated protein 1A/1B light chain 3; OD, optical density; 3MA, 3-methyladenine.

inhibited (Fig. 4C and D). Furthermore, a decrease in the number of GFP-LC3 dots also demonstrated that autophagy was inhibited (Fig. 4E). Therefore, it may be concluded that inhibition of autophagy by 3MA decreased the protective effect of FPS against VSMC calcification.

\section{Discussion}

Consistent with the observed calcification-inducing effect of Ox-LDL reported previously $(7,21)$, the present study demonstrated that Ox-LDL increased human VSMC calcification in a concentration-dependent manner, as determined by Alizarin Red S staining and measurement of $C B A F 1$ and $S M 22 \alpha$ mRNA expression levels by RT-qPCR. In addition, FPS treatment attenuated Ox-LDL-induced calcification of human VSMCs and alleviated the Ox-LDL-induced downregulation of autophagy. Furthermore, inhibition of autophagic activity by 3 MA decreased the protective effect of FPS against VSMC calcification. Therefore, to the best of the authors' knowledge, the present study is the first to demonstrate that FPS attenuates Ox-LDL-induced human VSMC calcification, at least partially, via upregulation of autophagy.
Vascular calcification is a pathological process whereby extraskeletal calcium-phosphate crystals are deposited in the vascular system (22). It is highly prevalent, as presented by the Multi-Ethnic Study of Atherosclerosis, in which the prevalence of coronary calcification in individuals of Caucasian, African-American, Hispanic and Chinese ethnicity was reported to be 70.4, 52.0, 56.6 and 59.2\%, respectively, for men, and 44.7, 37.0, 34.8 and 41.9\%, respectively, for women (23). Vascular calcification is a key risk factor for numerous adverse clinical outcomes and, during its development, a phenotypic change in VSMCs serves a critical role $(24,25)$. VSMCs undergo a phenotypic change from a contractile to a synthetic and osteochondrogenic phenotype, which is accompanied by downregulation of contractile markers expression, such as $S M 22 \alpha$, and upregulation of osteochondrogenic markers expression, such as CBFAl (26).

Oxidative stress has been implicated in vascular calcification, and has been demonstrated to promote VSMC differentiation (27). Liu et al (28) demonstrated that hydrogen peroxide enhanced vascular calcification by inducing osteoblastic differentiation of VSMCs. In our previous research, it was demonstrated that Ox-LDL promotes the osteogenic 
differentiation and calcification of human VSMCs $(21,29)$. In the present study, Ox-LDL treatment was illustrated to increase human VSMC mineralization, as indicated by Alizarin Red S staining, and significantly downregulated $S M 22 \alpha$ mRNA expression and upregulated $C B F A 1$ mRNA expression. These results confirmed that Ox-LDL increased human VSMC calcification.

Autophagy is a dynamic and highly regulated process of self-digestion, which is responsible for cell survival and response to oxidative stress (30). It is demonstrated to limit VSMC calcification by inhibiting matrix vesicle release (31). In an in vitro phosphate-induced calcification model of VSMCs and an in vivo model of chronic renal failure, it was reported that valproic acid-induced autophagy could decrease calcification, and that the inhibition of autophagy by 3MA could significantly promote phosphate-induced matrix vesicle release (32). In addition, it was reported that atorvastatin protected VSMCs from transforming growth factor $\beta 1$-stimulated calcification by inducing autophagy (33). Furthermore, a previous study illustrated that ghrelin treatment attenuated the elevation of calcium deposition in vascular calcification models in vivo and in vitro. Simultaneously, protein levels of the autophagy markers LC3 and beclin-1 were significantly upregulated by ghrelin in the vascular calcification model, and inhibition of autophagy by 3MA blocked the ameliorative effect of ghrelin on vascular calcification (34). From these results, it can be hypothesized that autophagy may be an endogenous protective mechanism counteracting vascular calcification, and that autophagy induction could be a therapeutic strategy for vascular calcification.

P62 accumulates when autophagy is inhibited, and is decreased when autophagy is induced; therefore, it can be used as a marker to study autophagic flux (35). Furthermore, during autophagy, the cytosolic form of LC3 (LC3-I) is conjugated to phosphatidylethanolamine to form an LC3-phosphatidylethanolamine conjugate (LC3-II), which is recruited to autophagosome membranes (36). Thus, the LC3-II/I ratio detected by western blot analysis is considered to be positively correlated with autophagic activity (37). In the present study, it was demonstrated that Ox-LDL treatment decreased the LC3-II/LC3-I ratio and simultaneously increased the P62 protein levels, suggesting that autophagic activity was inhibited under Ox-LDL stressor activity.

Cell injury induced by Ox-LDL is a major contributing factor to the pathogenesis of many cardiovascular diseases, and the accompanying inhibition of autophagy can be detected. For example, it was reported that in an Ox-LDL-induced model of human umbilical vein endothelial cell injury, the number of autophagosomes and the expression levels of autophagy-associated proteins were decreased (38). In an Ox-LDL-induced RAW264.7 cell injury model, autophagic activity was also presented to be inhibited, as confirmed by immunofluorescence, western blotting and monodansylcadaverine staining (39). Taken together, these results highlight that any treatment promoting autophagy may be considered to have a therapeutic potential in the treatment of Ox-LDL-associated injury.

FPS is a water-soluble polysaccharide that has previously been isolated from Fuzi (13). The total sugar content of the crude polysaccharide is determined to be $97.1 \%$, with a purity of $>99.8 \%$ (13). Previously, it was demonstrated that FPS protected against starvation-induced cytotoxicity in $\mathrm{H} 9 \mathrm{c} 2$ cells by increasing autophagy (15). Thus, in the present study, it was aimed to investigate whether FPS treatment could attenuate Ox-LDL-induced human VSMC calcification, and what role autophagy serves in the protective effects of FPS. Different concentrations of FPS were added to assess its effect on Ox-LDL-induced vascular calcification. It was demonstrated that FPS treatment attenuated the Ox-LDL-induced increase in Alizarin Red S staining in human VSMCs, indicating the amelioration of vascular calcification. Furthermore, the changes in $C B F A 1$ and $S M 22 \alpha$ mRNA levels induced by Ox-LDL were likewise reversed, further suggesting that FPS serves a protective role against $\mathrm{Ox}-\mathrm{LDL}$-induced vascular calcification.

Ox-LDL treatment decreased the LC3-II/LC3-I ratio and simultaneously increased the P62 protein levels, suggesting that autophagic activity was inhibited. Importantly, treatment with $1,000 \mu \mathrm{g} / \mathrm{ml}$ FPS could attenuate the aforementioned changes in the LC3-II/LC3-I ratio and P62 protein levels caused by Ox-LDL, suggesting that FPS treatment attenuated the Ox-LDL-induced downregulation of autophagic activity. This was also confirmed by the number of GFP-LC3 dots, which were used to monitor the number of autophagosomes. Subsequently, VSMCs were treated with 3MA, a commonly used pharmacological inhibitor of autophagy, and the effects on FPS-mediated protection against vascular calcification were observed. The addition of 3MA resulted in the inhibition of FPS protection. The autophagy changes indicated by the LC3-II/LC3-I ratio, P62 protein intensity and GFP-LC3 dots suggested that autophagy inhibition by $3 \mathrm{MA}$ decreased the protective effect of FPS against calcification.

In conclusion, the present study demonstrated for the first time, to the best of the authors' knowledge that FPS protects against Ox-LDL-induced vascular calcification in human VSMCs, likely by activating autophagy activity. It is possible that FPS can be developed into a novel type of medicine for vascular calcification treatment.

\section{Acknowledgements}

The present study was supported by the Natural Science Foundation of Guangdong Province, China (grant nos. 2015A030310185 and 2015A030313582), the Science and Technology Foundation of Guangdong Province, China (grant no. 2013B022000094) and the Natural Science Fund of China (grant no. 81701378).

\section{References}

1. Albanese I, Daskalopoulou SS, Yu B, You Z, Genest J, Alsheikh-Ali A and Schwertani AG: The Urotensin II system and carotid atherosclerosis: A role in vascular calcification. Front Pharmacol 7: 149, 2016.

2. Yahagi K, Kolodgie FD, Lutter C, Mori H, Romero ME, Finn AV and Virmani R: Pathology of human coronary and carotid artery atherosclerosis and vascular calcification in diabetes mellitus. Arterioscler Thromb Vasc Biol 37: 191-204, 2017.

3. Shigematsu T, Sonou T, Ohya M, Yokoyama K, Yoshida H, Yokoo T, Okuda K, Masumoto AR, Iwashita Y, Iseki K, et al: Preventive strategies for vascular calcification in patients with chronic kidney disease. Contrib Nephrol 189: 169-177, 2017.

4. Leopold JA: Vascular calcification: Mechanisms of vascular smooth muscle cell calcification. Trends Cardiovasc Med 25: 267-274, 2015.

5. Han X, Wang LY, Diao ZL and Liu WH: Apelin: A novel inhibitor of vascular calcification in chronic kidney disease. Atherosclerosis 244: 1-8, 2016. 
6. Zhang J, Chang JR, Duan XH, Yu YR and Zhang BH: Erratum to: Thyroid hormone attenuates vascular calcification induced by vitamin D3 plus nicotine in rats. Calcif Tissue Int 96: 580, 2015.

7. Tang Y, Xu Q, Peng H, Liu Z, Yang T, Yu Z, Cheng G, Li X, Zhang $G$ and Shi R: The role of vascular peroxidase 1 in ox-LDL-induced vascular smooth muscle cell calcification. Atherosclerosis 243: 357-363, 2015.

8. Ogawa S: Pathological mechanism of vascular calcification and new development in clinical strategy for the therapy. Clin Calcium 25: 633, 2015 (In Japanese).

9. Xue Z, Yuan W, Li J, Zhou H, Xu L, Weng J, Li X, Zhang X, Wang $Z$ and Yan J: Cyclophilin A mediates the ox-LDL-induced activation and apoptosis of macrophages via autophagy. Int J Cardiol 230: 142-148, 2017.

10. Yang J, Yu J, Li D, Yu S, Ke J, Wang L, Wang Y, Qiu Y, Gao X, Zhang J and Huang L: Store-operated calcium entry-activated autophagy protects EPC proliferation via the CAMKK2-MTOR pathway in ox-LDL exposure. Autophagy 13: 82-98, 2017.

11. Krishnamurthy M, Selvaraju M and Tamilarasan M: Turbinaria conoides (J. Agardh) sulfated polysaccharide protects rat's heart against myocardial injury. Int J Biol Macromol 50: 1275-1279, 2012.

12. Silva AK, Juenet M, Meddahi-Pelle A and Letourneur D Polysaccharide-based strategies for heart tissue engineering. Carbohydr Polym 116: 267-277, 2015

13. Zhao C, Li M, Luo Y and $\mathrm{Wu} \mathrm{W}$ : Isolation and structural characterization of an immunostimulating polysaccharide from fuzi, Aconitum carmichaeli. Carbohydr Res 341: 485-491, 2006.

14. Lin S, Liu K, Wu W, Chen C, Wang Z and Zhang X: Study on pretreatment of FPS-1 in rats with hepatic ischemia-reperfusion injury. Am J Chin Med 37: 323-337, 2009.

15. Liao LZ, Chen YL, Lu LH, Zhao YH, Guo HL and Wu WK: Polysaccharide from Fuzi likely protects against starvation-induced cytotoxicity in $\mathrm{H} 9 \mathrm{c} 2$ cells by increasing autophagy through activation of the AMPK/mTOR pathway. Am J Chin Med 41: 353-367, 2013.

16. Patel JJ, Srivastava S and Siow RC: Isolation, culture, and characterization of vascular smooth muscle cells. Methods Mol Biol 1430: 91-105, 2016

17. Gregory CA, Gunn WG, Peister A and Prockop DJ: An Alizarin red-based assay of mineralization by adherent cells in culture: Comparison with cetylpyridinium chloride extraction. Ana Biochem 329: 77-84, 2004.

18. Zhu D, Mackenzie NC, Shanahan CM, Shroff RC, Farquharson C and MacRae VE: BMP-9 regulates the osteoblastic differentiation and calcification of vascular smooth muscle cells through an ALK1 mediated pathway. J Cell Mol Med 19: 165-174, 2015 .

19. Livak KJ and Schmittgen TD: Analysis of relative gene expression data using real-time quantitative PCR and the 2(-Delta Delta C(T)) method. Methods 25: 402-408, 2001

20. Zhang Z, Singh R and Aschner M: Methods for the detection of autophagy in mammalian cells. Curr Protoc Toxicol 69: 20.12.1-20.12.26, 2016.

21. Song Y, Hou M, Li Z, Luo C, Ou JS, Yu H, Yan J and Lu L: TLR4/NF- $\kappa \mathrm{B} /$ Ceramide signaling contributes to Ox-LDL-induced calcification of human vascular smooth muscle cells. Eur J Pharmacol 794: 45-51, 2017.

22. Gao J, Zhang K, Chen J, Wang MH, Wang J, Liu P and Huang H: Roles of aldosterone in vascular calcification: An update. Eur J Pharmacol 786: 186-193, 2016.

23. Bild DE, Detrano R, Peterson D, Guerci A, Liu K, Shahar E, Ouyang P, Jackson S and Saad MF: Ethnic differences in coronary calcification: The Multi-Ethnic Study of Atherosclerosis (MESA). Circulation 111: 1313-1320, 2005
24. Zhao YG, Meng FX, Li BW, Sheng YM, Liu MM, Wang B, Li HW and Xiu RJ: Gelatinases promote calcification of vascular smooth muscle cells by up-regulating bone morphogenetic protein-2. Biochem Biophys Res Commun 470: 287-293, 2016.

25. Chen T, Mao H, Chen C, Wu L, Wang N, Zhao X, Qian J and Xing C: The Role and Mechanism of $\alpha$-Klotho in the calcification of rat aortic vascular smooth muscle cells. Biomed Res Int 2015: $194362,2015$.

26. Shanahan CM, Crouthamel MH, Kapustin A and Giachelli CM: Arterial calcification in chronic kidney disease: Key roles for calcium and phosphate. Circ Res 109: 697-711, 2011.

27. Cui L, Li Z, Chang X, Cong G and Hao L: Quercetin attenuates vascular calcification by inhibiting oxidative stress and mitochondrial fission. Vascul Pharmacol 88: 21-29, 2017.

28. Liu H, Lu Q and Huang K: Selenium suppressed hydrogen peroxide-induced vascular smooth muscle cells calcification through inhibiting oxidative stress and ERK activation. J Cell Biochem 111: 1556-1564, 2010.

29. Liao L, Zhou Q, Song Y, Wu W, Yu H, Wang S, Chen Y, Ye M and $\mathrm{Lu} \mathrm{L}$ : Ceramide mediates Ox-LDL-induced human vascular smooth muscle cell calcification via p38 mitogen-activated protein kinase signaling. PLoS One 8: e82379, 2013.

30. Yu L, Chen Y and Tooze SA: Autophagy pathway: Cellular and molecular mechanisms. Autophagy: Sep 21, 2017 (Epub ahead of print).

31. Shanahan CM: Autophagy and matrix vesicles: New partners in vascular calcification. Kidney Int 83: 984-986, 2013.

32. Dai XY, Zhao MM, Cai Y, Guan QC, Zhao Y, Guan Y, Kong W, Zhu WG, Xu MJ and Wang X: Phosphate-induced autophagy counteracts vascular calcification by reducing matrix vesicle release. Kidney Int 83: 1042-1051, 2013.

33. Liu D, Cui W, Liu B, Hu H, Liu J, Xie R, Yang X, Gu G, Zhang J and Zheng $\mathrm{H}$ : Atorvastatin protects vascular smooth muscle cells from TGF- $\beta 1$-stimulated calcification by inducing autophagy via suppression of the $\beta$-catenin pathway. Cell Physiol Biochem 33: 129-141, 2014.

34. Xu M, Liu L, Song C, Chen W and Gui S: Ghrelin improves vascular autophagy in rats with vascular calcification. Life Sci 179: 23-29, 2017

35. BenYounès A, Tajeddine N, Tailler M, Malik SA, Shen S, Métivier D, Kepp O, Vitale I, Maiuri MC and Kroemer G: A fluorescence-microscopic and cytofluorometric system for monitoring the turnover of the autophagic substrate p62/SQSTM1. Autophagy 7: 883-891, 2011.

36. Schläfli AM, Adams O, Galván JA, Gugger M, Savic S, Bubendorf L, Schmid RA, Becker KF, Tschan MP, Langer R and Berezowska S: Prognostic value of the autophagy markers LC3 and p62/SQSTM1 in early-stage non-small cell lung cancer. Oncotarget 7: 39544-39555, 2016.

37. Tanida I, Ueno T and Uchiyama Y: A super-ecliptic, pHluorinmKate2, tandem fluorescent protein-tagged human LC3 for the monitoring of mammalian autophagy. PLoS One 9: e110600, 2014.

38. Luo Y, Meng X, Zhou P, Lu S, Qin M, Xu X, Sun G and Sun X: Elatoside $\mathrm{C}$ protects against ox-LDL-induced HUVECs injury by FoxO1-mediated autophagy induction. Biochim Biophys Acta 1863: 1654-1665, 2017.

39. Zhang BC, Zhang CW, Wang C, Pan DF, Xu TD and Li DY: Luteolin attenuates foam cell formation and apoptosis in Ox-LDL-stimulated macrophages by enhancing autophagy. Cell Physiol Biochem 39: 2065-2076, 2016.

This work is licensed under a Creative Commons Attribution-NonCommercial-NoDerivatives 4.0 International (CC BY-NC-ND 4.0) License. 